\title{
Design and Evaluation of a System for Microscope-Assisted Guided Interventions (MAGI)
}

\author{
Philip J. Edwards ${ }^{1}$, Andrew P. King ${ }^{1}$, Calvin R. Maurer, Jr. ${ }^{1}$, \\ Darryl A. de Cunha ${ }^{1}$, David J. Hawkes ${ }^{1}$, Derek L. G. Hill ${ }^{1}$, \\ Ron P. Gaston ${ }^{1}$, Michael R. Fenlon ${ }^{2}$, Subhash Chandra ${ }^{3}$, Anthony J. Strong ${ }^{4}$, \\ Christopher L. Chandler ${ }^{4}$, Aurelia Richards ${ }^{5}$, and Michael J. Gleeson ${ }^{5}$ \\ 1 Computational Imaging Science Group (CISG) \\ Radiological Sciences, KCL, Guy's Hospital, London, UK \\ 2 Department of Prosthetic Dentistry, Guy's Hospital, London, UK \\ ${ }^{3}$ Leica, USA \\ 4 Neurosurgery Dept., King's College Hospital, London, UK \\ 5 ENT surgery dept., Guy's Hospital, London, UK
}

\begin{abstract}
The problem of providing surgical navigation using image overlays on the operative scene can be split into four main tasks - calibration of the optical system; registration of preoperative images to the patient; tracking of the display system and patient and display using a suitable visualisation scheme.

To achieve a convincing result in the magnified view through the operating microscope high alignment accuracy is required. We have simulated our entire system to establish the major sources of error. We have improved each of the stages involved. The microscope calibration process has been automated. We have introduced bone-implanted markers for registration and incorporated a locking acrylic dental stent (LADS) for patient tracking and/or registration.

These improvements have significantly increased the alignment accuracy of our overlays. LADS repositioning on volunteers showed a mean target registration error of $0.7 \mathrm{~mm}$. Phantom accuracy is $0.3-0.5 \mathrm{~mm}$ and clinical overlay errors were $0.5-1.0 \mathrm{~mm}$ on the bone fiducials and $0.5-4 \mathrm{~mm}$ on target structures. We have improved the graphical representation of the stereo overlays. The resulting system provides $3 \mathrm{D}$ surgical navigation for microscope-assisted guided interventions (MAGI).
\end{abstract}

\section{Introduction}

In conventional image-guided surgery a $3 \mathrm{D}$ representation of the patient is generated, usually from MR or CT images, prior to surgery. At surgery correspondence between this representation and the patient is established by registration. A computer displays the location in this representation of a pointer held within the surgical field. Use of such a system relies on the surgeon looking away from the surgical scene to the computer workstation. This is not ideal as it requires mental reorientation between the surgical view and the workstation display. 
A solution that we are pursuing is to overlay $3 \mathrm{D}$ projections derived from the preoperative images, into the binocular optics of a surgical microscope, accurately aligned with the surgical scene. The microscope optics are of high quality and provide an ideal device for incorporation of an augmented reality display. Proof of principle has been presented [1] and was inspired by the earlier work of Kelly and Roberts [2,3]. To exploit fully the potential of this technology in microsurgery it has become clear that registration between overlay and surgical scene must be as accurate as possible.

In the following sections we describe the equipment and methods of calibration, registration, tracking and visualisation. An error analysis of the system is given along with the results of volunteer, phantom and clinical experiments.

\section{Method}

\section{$2.1 \quad$ Equipment}

A Leica M695 or M500 binocular operating microscope with an interface to record focus and zoom settings is adapted by incorporating two purpose built monochrome VGA 640x480 displays. These project overlay images into the left and right views via beam-splitters. A video camera system records the combined overlays and microscope views. Changes in the position of the microscope are tracked using an array of 13 infra-red emitting diodes (IREDs) mounted on a bracket which is rigidly attached to the microscope assembly. The 3D IRED coordinates are measured using an Optotrak localiser (Northern Digital Inc.).

For registration we use fiducials that are based on the bone-implanted marker designs of Maurer et al [4]. A post is screwed into the skull. Attached to this post is either an imaging marker or a physical locator, the centres of which coincide.

Changes in patient position are determined by tracking the position of 6 IREDs fixed to the custom built locking acrylic dental stent (LADS). The LADS assembly also contains 10 fiducials which are the same as those implanted in the bone and may be used for registration.

The main user interface and preoperative image display system runs on a Sun sparcstation. This communicates with an Intergraph graphics PC which provides the two overlay images injected into each eyepiece of the microscope.

\subsection{Calibration}

In order to produce good alignment of the overlays with the real world, accurate calibration of the microscope optics is necessary. We assume a pinhole camera model and neglect distortion, which we have measured to be only $1-2 \%$ at the extreme edge of the field-of-view.

We employ an automated calibration method which uses the calibration object shown in Figure 1(a). This object is fitted with a set of IREDs and tracked whilst the calibration images are acquired by a camera mounted inside the microscope. The calibration object has a known pattern of circular markings which 
are automatically detected and matched. A calibration image is shown in Figure $1(\mathrm{~b})$. The matching information is combined with the tracking data to produce a set of corresponding $3 \mathrm{D}$ and $2 \mathrm{D}$ points, which can be used to calibrate the microscope optics. For each calibration, a number of images are acquired from different relative positions of the microscope and calibration object. This increases the number of point correspondences used in the calibration; reduces any random errors in tracking the microscope; and provides a better spread of calibration points along the optical axis. The Tsai calibration algorithm[5] is used to obtain estimates of the camera parameters. This procedure is carried out separately for both eye-pieces of the microscope.

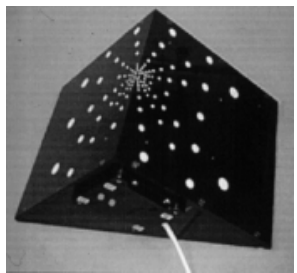

(a)

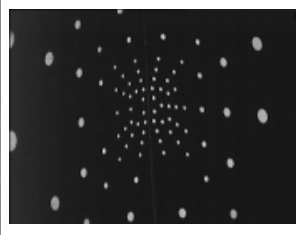

(b)

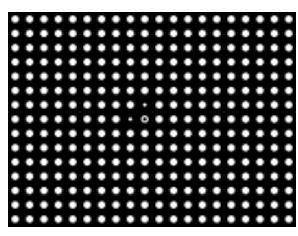

(c)

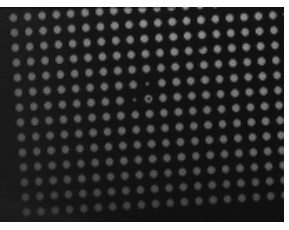

(d)

Fig. 1. The calibration object and 2D calibration pattern: (a) perspective view of the calibration object showing the IREDs used for tracking, (b) an image of the calibration object acquired by the microscope camera, (c) the $2 \mathrm{D}$ calibration pattern, (d) an image of the $2 \mathrm{D}$ calibration pattern acquired by the microscope camera

We calibrate over the range of variable zoom and focus parameters. This is achieved by modelling the variation in the values of the camera parameters as bivariate polynomial functions of zoom and focus $[6,7]$.

Currently there are two modes of operation of the MAGI system: variable zoom and focus calibrations are useful when a range of zoom and focus settings will be used in a single procedure, whereas fixed zoom and focus calibrations are used when we have prior knowledge of the required zoom and focus settings.

Alignment of the coordinate systems of the microscope camera and the image injectors is achieved by injecting an image of a calibration pattern into each microscope eye-piece, and automatically matching it with the known model. The pattern used is shown in Figure 1(c) and an image acquired through the microscope camera is shown in Figure 1(d). The circles in the calibration pattern can be accurately localised using a centre of gravity operator, so the 2D overlay calibration mapping can be done with sub-pixel accuracy. 


\subsection{Image-to-Physical Registration and Patient Tracking}

For registration, the application accuracy for skin markers or anatomical landmarks is typically about $3 \mathrm{~mm}$ or worse [8], whereas bone-implanted markers inserted prior to preoperative imaging give a clinical accuracy of $\sim 0.7 \mathrm{~mm}[4]$. We have produced markers based on this system. We can mark the physical caps with a tracked pointer to an accuracy of $\sim 0.2 \mathrm{~mm}$. The image markers can be found repeatably to an accuracy of $\sim 0.4 \mathrm{~mm}$ in MRI images of voxel size $1 \times 1 \times 1.5 \mathrm{~mm}$. Taking a gradient echo MRI scan we get typical fiducial registration errors of $0.7-1.2 \mathrm{~mm}$ due to imager distortion.

A tracker needs to be rigidly attached to the skull to track movement of the patient. In conventional systems the head is immobilised in a Mayfield or similar clamp to which a tracker is fixed. Since we are interested in both ENT and neurosurgical applications a more convenient tracking system is required. We have designed a locking acrylic dental stent (LADS) which attaches to the patient's upper teeth. This enables much freer movement of the patient's head.

Dental appliances such as occlusal splints and bite blocks have been proposed for use as positional reference devices $[9,10]$. For our purposes the standard occlusal splint was redesigned to maximise retention and stability with the aim of improved repeatability of placement. The main occlusal block is clamped with titanium screws to left and right locking wings. It is possible that the need to insert markers into the skull can be avoided by attaching the fiducials to the LADS. For this purpose we have designed a set of extended arm pieces to which imaging and physical locators can be attached. The LADS is shown in figure 2 .

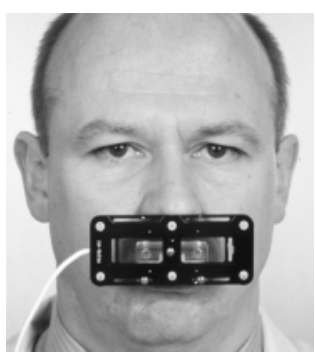

(a)

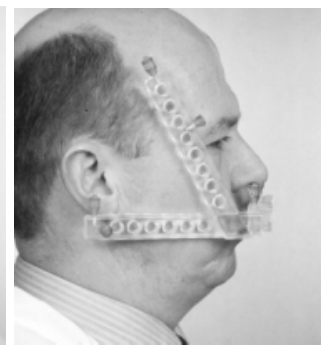

(b)

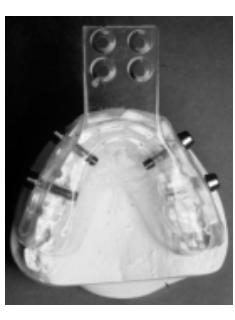

(c)

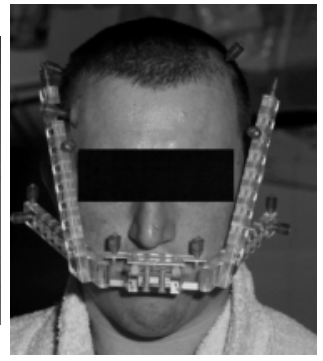

(d)

Fig. 2. Registration methods. The locking acrylic dental stent (LADS) construction, showing (a) the optical tracker attachment, (b) the image and physical fiducial frame, (c) the acrylic stent with locking screws and (d) a patient with both the LADS marker frame and bon-implanted fiducials.

To assess the suitability of the LADS for registration we need to measure how accurately the stent can be relocated in the same position. For this purpose we have scanned four volunteers with the LADS device in place. Multiple scans are 
taken and the LADS is removed and replaced between each scan. The scans are registered to each other using the fiducials on the LADS frame. This transformation is compared with that obtained by fully automated registration of each pair of MR images by maximisation of Normalised Mutual Information [11]. The transformations incorporate 3 extra degrees of freedom to compensate for scaling inaccuracy along the axes of the MR scanner.

\subsection{Visualisation}

Accurate calibration of each eye should give the correct stereo disparity for overlaid objects. For true 3D image guidance it is necessary to provide correct 3D perception of the position of overlaid objects. To this end we have conducted, both in a laboratory and through the microscope itself, a number of vision experiments to establish the optimum parameters for 3D stereo perception through transparent surfaces. Preliminary results show that the overlay should be carefully controlled and provide maximum colour separation from the surgical scene. The detail should include identifiable regions with different spatial frequencies. A reasonable approximation to this is provided by a wireframe representation.

\subsection{Error Analysis}

We have undertaken a numerical simulation of the MAGI system, which enables the levels of the various noise sources to be varied individually and their effect on the overall system error observed. The simulation is achieved by taking a typical configuration of IREDs, fiducials and calibration points and adding Gaussian noise of a similar size to the relevant measurement error - Optotrak IRED tracking, fiducial identification in the images and calibration marker localisation in the camera image. All the necessary processing (calibration, registration and rigid body tracking of each object) is performed using both noisy and exact values the comparison of these two gives us the simulated error. This was repeated 1000 times to produce the error statistics, with a set of 500 random points within the surgical field being used to compute target errors. The values of interest are calibration error, error in the 3D location of virtual structures, and the corresponding $2 \mathrm{D}$ overlay error after projection onto the image plane.

\subsection{Phantom Experiments and Initial Clinical Evaluation}

The entire system has been tested on a phantom and in the operating theatre. Overlay accuracy is assessed by identifying corresponding real and virtual structures in grabbed images. This gives a 2D overlay error which has a corresponding $3 \mathrm{D}$ error in $\mathrm{mm}$ at the focal plane.

\section{Results}

\subsection{Simulation Results}

The sources of error in the MAGI system can be split into three categories: error in localising the circular markings in the calibration images; error in localising 
the bone-implanted imaging markers in the preoperative image; and tracking error. For each of these we estimate typical error values. For localising the calibration object marks with a centre of gravity operator we estimate an error of 1 pixel RMS. For a typical CT and MRI slice thickness and in-plane resolution, and using a centre-of-gravity operator to obtain sub-voxel accuracy in the preoperative image, we estimate the imaging marker localisation error to be $0.4 \mathrm{~mm}$ RMS (neglecting MR distortion). Finally, careful experiments have confirmed that the Optotrak tracks individual IREDs to an accuracy of $0.2 \mathrm{~mm}$ RMS.

Table 1. The effects of individual error sources on the overall system error. Tracking errors are broken down by tracked object. A final system error is predicted. Typical error values are used for the three error sources: 1 pixel RMS for calibration mark localisation; $0.4 \mathrm{~mm}$ RMS for imaging marker localisation; and $0.2 \mathrm{~mm}$ RMS for tracking.

\begin{tabular}{|l|c|c||c|}
\hline $\begin{array}{l}\text { Error } \\
\text { Source }\end{array}$ & $\begin{array}{c}\text { Calibration Error } \\
(\mathrm{mm} \text { at focal plane })\end{array}$ & $\begin{array}{c}\text { DD Alignment } \\
\text { Error }(\mathrm{mm})\end{array}$ & $\begin{array}{c}\text { 2D Overlay Error } \\
(\mathrm{mm} \text { at focal plane })\end{array}$ \\
\hline \hline Calibration marks & 0.014 & - & 0.014 \\
\hline \hline Imaging markers & - & 1.1 & 0.55 \\
\hline \hline Tracking & 0.29 & 1.2 & 0.71 \\
\hline Calibration object & 0.060 & - & 0.060 \\
\hline Microscope & 0.28 & 0.43 & 0.45 \\
\hline LADS & - & 0.86 & 0.41 \\
\hline Pointer & - & 0.69 & 0.30 \\
\hline \hline Combined system error & 0.26 & 1.6 & 0.91 \\
\hline
\end{tabular}

Using these estimates, Table 1 shows the relative importance of the 3 error sources. Whilst errors in localising the imaging markers are significant, tracking errors are the most important error source. For this reason these are broken down further. Four objects are tracked in the current system configuration: the calibration object, the localisation pointer, the LADS and the microscope. Using the estimated tracking error of $0.2 \mathrm{~mm}$ RMS, Table 1 shows the relative importance of errors in tracking each of these four objects. As can be seen, microscope tracking is the most important factor in the final 2D overlay error. This is because it contributes both to the calibration error and the intraoperative errors. Also, the area of interest (i.e. the surgical scene) is relatively far from the microscope, so rotational errors will have a greater effect. Tracking of the patient (LADS) and the localisation pointer also have a significant effect, in particular on the 3D alignment error. Combining all of these error sources, and using the same estimated typical error values, we get an estimate of the final overall accuracy of the MAGI system. The total error prediction is $0.91 \mathrm{~mm}$ at the focal plane of the microscope for a typical intraoperative marker configuration. This figure is reduced to $0.6 \mathrm{~mm}$ for the idealised phantom marker positions. 
Calibration of Zoom and Focus The simulation suggests that the error of the automatic fixed zoom and focus calibration technique is approximately $0.26 \mathrm{~mm}$. This is consistent with observed calibration accuracies. Further errors will be introduced from the modelling of the perspective projection parameters with zoom and focus. After this modelling the average calibration error over all possible zoom and focus values is $0.3-0.4 \mathrm{~mm}$. The error rises for minimum zoom and far focus due to inadequate modelling with the bivariate polynomial in this region. At these settings a fixed zoom and focus calibration is used.

\subsection{LADS Relocation Accuracy}

All image pairs for a given volunteer were registered using the LADS fiducials and the automated method [11]. The target registration error (TRE) was computed as the Euclidean distance between all voxels within the head transformed with the automated registration and that derived from the LADS fiducials. Table 2 gives the results. The mean TRE over all datasets was $0.7 \mathrm{~mm}$ with a maximum TRE over all the data of $3.64 \mathrm{~mm}$. Registration accuracy is best around the oral and nasal region, where the error produced by the relocation of the LADS assembly is comparable to that using the bone-implanted markers.

Table 2. LADS target registration accuracy for four volunteers

\begin{tabular}{|c||c|c||c|}
\hline \multirow{2}{*}{ Volunteer } & \multicolumn{2}{|c||}{ mean TRE } & \multirow{2}{*}{ max TRE } \\
\cline { 2 - 3 } & mean & stddev & \\
\hline $\mathrm{A}$ & 0.69 & 0.22 & 1.82 \\
\hline $\mathrm{B}$ & 0.57 & 0.18 & 1.44 \\
\hline $\mathrm{C}$ & 1.14 & 0.38 & 3.64 \\
\hline $\mathrm{D}$ & 0.60 & 0.17 & 2.19 \\
\hline Combined & 0.70 & 0.30 & 3.64 \\
\hline
\end{tabular}

\subsection{Phantom and Clinical Results}

Incomplete versions of the system have been evaluated on 6 patients. The full system with stereo graphics for the overlays and the latest version of the LADS has been used on two patients described below. Overlays on the phantom have been measured to have errors of $0.3-0.5 \mathrm{~mm}$, slightly less than the predicted $0.6 \mathrm{~mm}$ error.

The first patient underwent removal of a petrous apex cyst. Since the patient had bilateral cysts a trans-labyrinth approach was not appropriate. A more anterior approach was used in order to preserve hearing. The LADS and bone fiducials were both used. One fiducial was marked at the start and end of the procedure, giving a positional error of $1.4 \mathrm{~mm}$. This is a small change indicating that the LADS was stable over the 8 hours of the operation. The alignment of 


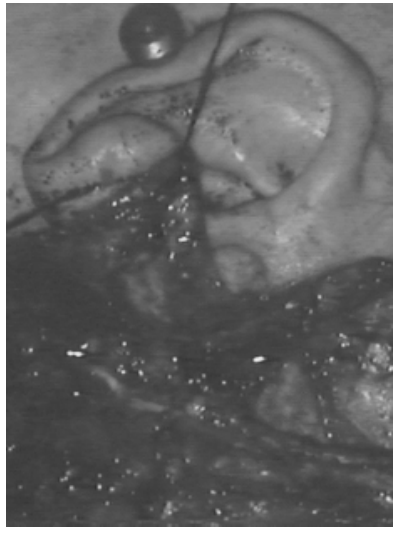

(a)

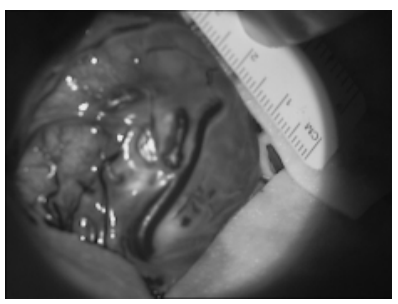

(d)

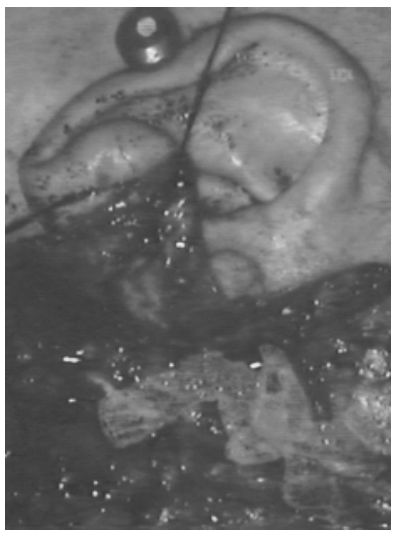

(b)

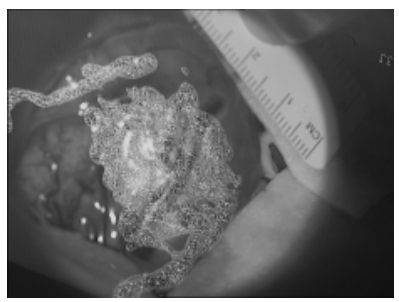

(e)

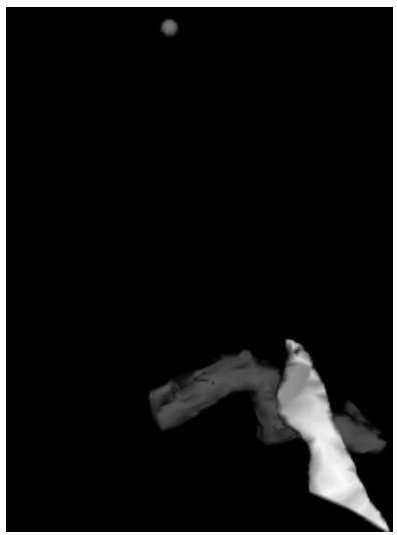

(c)

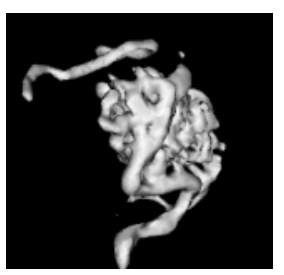

(f)

Fig. 3. Clinical results for petrous apex cyst patient (a) the microscope view, (b) the same view with overlay of zygomatic arch, carotid artery and bone pin and (c) a rendering from a similar viewpoint; results for a patient with an AVM showing (d) the microscope view, (e) the same view with overlay of the AVM and (f) a rendering from a similar viewpoint.

the overlays was visually accurate (see figure 3 ) to $0.5-1.0 \mathrm{~mm}$ on the registration pins and better than $1 \mathrm{~mm}$ for the zygomatic arch.

To our knowledge this is the first operation guided by microscope overlays providing good 3D overlay perception by stereo. The feedback from the surgeon was very positive. He remarked that the system had improved confidence in this unusual approach. The cyst was removed and the patient's hearing preserved.

For the second patient only the LADS was used for registration. The patient had a small arteriovenous malformation (AVM) in the parietal lobe between the motor and sensory strips. The system was used to provide an overlay of the lesion before craniotomy and influenced the craniotomy site chosen. The surgeon remarked that this had been helpful. The overlay on the microscope view of the brain surface is shown in figure 3 . We estimate the displacement to be $3-4 \mathrm{~mm}$, 
the brain surface having sagged in the 40 minutes since the dura was opened. Such deformation has been observed as a common occurrence in neurosurgical procedures $[12,13]$.

For the results above a fixed zoom and focus calibration at maximum focal length and minimum zoom was used to give the easiest depth perception.

\section{Discussion}

It became clear in early clinical evaluations of the initial system [1] that improved accuracy in the operating theatre was essential. We have achieved this through investigation of errors and a number of improvements to the system.

Automated calibration has provided us with a microscope calibration accuracy of $0.3-0.4 \mathrm{~mm}$ for the practical range of zoom and focus values. An error simulation of the entire system has been implemented which can be used to experiment with alternative configurations. An overall system error of $1.6 \mathrm{~mm}$ in $3 \mathrm{D}$ and $0.91 \mathrm{~mm}$ in the $2 \mathrm{D}$ overlays is predicted. As a result of experiments with the simulation we intend to add IREDs to the microscope frame, and also possibly the LADS tracker, with greater separation to reduce the tracking error. To reduce registration error we have incorporated bone-implanted fiducials.

For patient tracking we introduce the locking acrylic dental stent (LADS). This is an extremely versatile tracker which allows free movement of the head within the line-of-sight of the Optotrak and enables image guidance in operations for which a head clamp is unacceptable. This is an important advancement which makes the MAGI system applicable to many more procedures. The LADS may also be a good registration device. Volunteer experiments are very encouraging and clinical accuracy will be measured in forthcoming cases. As well as providing a non-invasive registration system the physical fiducial localisation can take place without the patient, which saves valuable time in the operating theatre.

For visualisation we have incorporated high speed graphics cards with wireframe rendering to improve stereo perception. Experiments are underway to establish the ideal visual parameters for perception of the virtual structures beneath the operative surface. This has been achieved through the microscope on a skull phantom, with structures being easily perceived in the correct position by the majority of people.

Phantom results showed accuracy of $0.3-0.5 \mathrm{~mm}$ and initial clinical results had errors of $0.5-4 \mathrm{~mm}$ on target structures. We will be continuing clinical trials of the system in which the accuracy of the registration and the overlays as well as the perception of depth will be assessed. The aim is to enable the surgeon to see structures beneath the operative surface as though the tissue were transparent. Initial results using the improved accuracy and visualisation capabilities of MAGI have been very encouraging. 


\section{Acknowledgements}

We would like to thank Leica and the EPSRC for funding this project through the DTI's MedLINK programme (project P108). We are also grateful to the radiology, radiography and theatre staff at Guy's and King's hospitals for their cooperation and to Prof. Rolf Hauser of the ENT surgery dept., Kantonsspital, Basel, Switzerland for advice on the development of the LADS device during his stay at Guy's hospital in 1997.

\section{References}

1. P. J. Edwards, D. J. Hawkes, D. L. G. Hill, D. Jewell, R Spink, A. J. Strong, and M. J. Gleeson. Augmentation of reality in the stereo operating microscope for otolaryngology and neurosurgical guidance. Computer Assisted Surgery (formerly Journal of Image Guided Surgery), 1(3):172-178, 1995. 843, 850

2. P. J. Kelly, G.J. Alker, and S. Goerss. Computer-assisted stereotactic laser microsurgery for the treatment of intracranial neoplasms. Neurosurgery, 10:324-331, 1982. 843

3. D. W. Roberts, J. W. Strohbehn, J.F. Hatch, W. Murray, and H. Kettenberger. A frameless stereotaxic integration of computerized tomographic imaging and the operating microscope. Journal of Neurosurgery, 65:545-549, 1986. 843

4. C. Maurer, M. J. Fitzpatrick, M. Y. Wang, R. L. Galloway, R. J. Maciunas, and G. S. Allen. Registration of head volume images using implantable fiducial markers. IEEE Transactions on Medical Imaging, 16(4):447-461, $1997 . \quad 843,845$

5. R. Y. Tsai. A versatile camera calibration technique for high-accuracy $3 \mathrm{~d}$ machine vision metrology using off-the-shelf tv cameras and lenses. IEEE Journal of Robotics and Automation, 3(4):323-344, 1987. 844

6. R. G. Wilsson. Modelling and Calibration of Automated Zoom Lenses. PhD thesis, The Robotics Institute, Carnegie Mellon University, 1994. 844

7. O. J. Fleig, P. J. Edwards, S. Chandra, H. Stüttler, and D.J. Hawkes. Automated microscope calibration for image guided surgery. In Proceedings of ComputerAssisted Radiology and Surgery 1998. Elsevier, Amsterdam, 1998. 844

8. J. P. Wadley, N.L. Dorward, M. Breeuwer, F.A. Gerritsen, N.D. Kitchen, and D. G. T. Thomas. Neuronavigation in 210 cases: further development of applications and full integration into contemporary neurosurgical practice. In Proceedings of Computer-Assisted Radiology and Surgery 1998, pages 635-640. Elsevier, Amsterdam, 1998. 845

9. M. A. Howard, M. B. Dobbs, T. M. Siminson, W. E. LaVelle, and M. A. Granner. A non-invasive reattachable skull fiducial marker system. Journal of Neurosurgery, 83:372-376, 1995. 845

10. R. Hauser, B. Westermann, and R. Probst. Non-invasive tracking of patients' head movements during computer-assisted intranasal microscopic surgery. Laryngoscope, 211:491-499, 1997. 845

11. C Studholme, D.L.G. Hill, and D.J. Hawkes. Automated 3-D registration of MR and CT images of the head. Medical Image Analysis, 1(2):163-175, 1996. 846, 848 
12. D. L. G. Hill, C. R. Maurer, R. J. Maciunas, J. M. Barwise, J. A. Fitzpatrick, and M. Y. Wang. Measurement of intraoperative brain surface deformation under a craniotomy. Neurosurgery, 43(3):514-528, 1998. 850

13. D. W. Roberts, A. Hartov, F. E. Kennedy, M. I. Miga, and K. D. Paulsen. Intraoperative brain shift and deformation: A quantitative analysis of cortical displacement in 28 cases. Neurosurgery, 43(4):749-760, 1998. 850 